\title{
Impacto da COVID-19 no controle e reorganização da atenção à tuberculose
}

Impact of COVID-19 on the control and reorganization of tuberculosis care Impacto de la COVID-19 en el control y reorganización de la atención a la tuberculosis

Paula Hino 1 (6) https://orcid.org/0000-0002-1408-196X

Thais Tiemi Yamamoto ${ }^{2}$ in https://orcid.org//0000-0002-7714-901x Gabriela Tavares Magnabosc $0^{3}$ @i nttps://orid. org/0000-0003-3318-6748

Maria Rita Bertolozzi ${ }^{4}$ io https://orcid.org/0000-0002-5009-5285

Mônica Taminato ${ }^{1}$ ị https://orcid.org//0000-0003-4075-2496

Lucimara Fabiana Fornari ${ }^{4}$ iD https://orcid.org/0000-0002-8655-6549

Como citar:

Hino P, Yamamoto TT, Magnabosco GT, Bertolozzi MR, Taminato M, Fornari LF. Impacto da COVID-19 no controle e reorganização da atenção à tuberculose. Acta Paul Enferm. 2021;34:AAPE002115.

DOI http://dx.doi.org/10.37689/actaape/2021AR02115

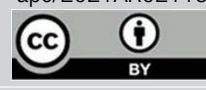

Descritores

Tuberculose; Coronavírus; COVID-19; Infecções por coronavirus; Saúde pública

Keywords

Tuberculosis; Coronavirus; COVID-19; Coronavirus infections; Public health

Descriptores

Tuberculose; Coronavirus; COVID-19; infeciones por coronavirus; Salud publica

Submetido

3 de Agosto de 2020

Aceito

31 de Maio de 2021

\section{Autora correspondente}

Paula Hino

E-mail: paulahino@yahoo.com.br

\section{Resumo}

Objetivo: Identificar as evidências científicas sobre 0 impacto da pandemia de COVID-19 na atenção e no controle da tuberculose.

Métodos: Revisão de escopo realizada em junho de 2020 nas seguintes bases de dados: Cochrane Library, Embase, LILACS, MEDLINE, PubMed, Web of Science, Cinahl e Scopus; e literatura cinzenta-Opengrey. Os descritores utilizados na busca foram: coronavirus, COVID-19, SARS-CoV-2 e tuberculosis. 0 processo de seleção foi feito por dois revisores independentes por meio da plataforma Rayyan, com a inclusão de 30 estudos.

Resultados: A pandemia da COVID-19 teve impacto no controle da tuberculose e os principais desafios decorrentes relacionam-se à influência do distanciamento social no diagnóstico, seguimento e adesão ao tratamento, incluindo a reorganização dos serviços de tuberculose, principalmente como resultado do necessário deslocamento de equipes de saúde que atuavam na área para a atenção à COVID-19, registrandose também limitação de acesso a insumos e a serviços de saúde, o que de igual modo ocorreu em relação a outros agravos de saúde, evidenciando-se vulnerabilidade programática. Destacam-se, ainda, os efeitos da pandemia na dimensão social, o que contribuiu para o aumento da vulnerabilidade social.

Conclusão: Muitos são os desafios impostos pela pandemia na COVID-19, particularmente no que diz respeito à manutenção das ações de controle da tuberculose. Espera-se que esta revisão contribua para embasar novos estudos e para a implementação de políticas públicas orientadas ao enfrentamento de ambas as enfermidades.

\section{Abstract}

Objective: To identify scientific evidence on the impact of the COVID-19 pandemic on tuberculosis care and control.

Methods: Scoping review conducted in June 2020 in the following databases: Cochrane Library, Embase, LILACS, MEDLINE, PubMed, Web of Science, Cinahl and Scopus; and Opengrey - grey literature. The descriptors used in the search were: coronavirus, COVID-19, SARS-CoV-2 and tuberculosis. The selection process was performed by two independent reviewers using the Rayyan platform with the inclusion of 30 studies.

Results: The COVID-19 pandemic had an impact on tuberculosis control and the main challenges arising from it are related to the influence of social distancing on diagnosis, follow-up and adherence to treatment, including the reorganization of tuberculosis services, mainly as a result of the necessary mobilization of health teams working in the area for the care of COVID-19. Limited access to inputs and health services was also

Escola Paulista de Enfermagem, Universidade Federal de São Paulo, São Paulo, SP, Brasil.

${ }^{2}$ Secretaria Municipal de Saúde, Coordenadoria de Vigilância em Saúde, São Paulo, SP, Brasil.

${ }^{3}$ Faculdade de Enfermagem, Universidade Estadual de Maringá, Maringá, PR, Brasil.

${ }^{4}$ Escola de Enfermagem, Universidade de São Paulo, São Paulo, SP, Brasil.

Conflitos de interesse: nada a declarar. 
registered, which occurred similarly in relation to other health problems, thereby showing programmatic vulnerability. The effects of the pandemic in the social dimension that contributed to increase the social vulnerability also stand out.

Conclusion: Many challenges have been posed by the COVID-19 pandemic, particularly with regard to maintaining tuberculosis control actions. We expect this review will contribute to support new studies and implement public policies aimed at confronting both diseases.

\section{Resumen}

Objetivo: Identificar las evidencias científicas sobre el impacto de la pandemia de COVID-19 en la atención y el control de la TB.

Métodos: Revisión de alcance realizada en junio de 2020 en las siguientes bases de datos: Cochrane Library, Embase, LILACS, MEDLINE, PubMed, Web of Science, Cinahl y Scopus, y literatura gris-Opengrey. Los descriptores utilizados en la búsqueda fueron: coronavirus, COVID-19, SARS-CoV-2 y tuberculosis. El proceso de selección fue realizado por dos revisores independientes a través de la plataforma Ryyan, con la inclusión de 30 estudios.

Resultados: La pandemia de COVID-19 tuvo impacto en el control de la tuberculosis y los principales desafíos derivados están relacionados con la influencia del distanciamiento social en el diagnóstico, seguimiento y adherencia al tratamiento, lo que incluye la reorganización de los servicios de TB, principalmente como resultado del necesario traslado de los equipos de salud que actuaban en el área hacia la atención a la COVID-19. También se registraron limitaciones de acceso a insumos y servicios de salud, lo que ocurrió de igual modo con relación a otros problemas de salud y dejó en evidencia la vulnerabilidad programática. Además, se observan efectos de la pandemia en la dimensión social, lo que contribuye al aumento de la vulnerabilidad social.

Conclusión: Muchos son los desafíos impuestos por la pandemia de COVID-19, particularmente en lo que se refiere a mantener las acciones de control de la tuberculosis. Se espera que esta revisión contribuya para fundamentar nuevos estudios y para la implementación de políticas públicas orientadas al afrontamiento de ambas enfermedades.

\section{Introdução}

A COVID-19, infecção causada pelo vírus SARsCoV-2, foi declarada pandemia em março de 2020 pela Organização Mundial de Saúde (OMS). ${ }^{(1)}$ Esse evento, de proporçôes catastróficas, tem afetado milhôes de famílias, com importantes repercussōes sociais, em especial em relação ao agravamento da desigualdade social, acentuando problemas globais de saúde pública, dentre eles, o controle da tuberculose (TB), que é a principal causa de morte por uma única doença infecciosa em todo o mundo. ${ }^{(2)}$

As pessoas com $\mathrm{TB}$ ou que tiveram a doença sáo consideradas vulneráveis à COVID-19, pois podem desenvolver formas mais graves da doença em decorrência do comprometimento pulmonar prévio. $\mathrm{O}$ diagnóstico precoce da TB e da COVID-19 pode evitar evoluçáo clínica desfavorável. ${ }^{(3)}$ Dada a rápida progressão da pandemia e a necessária adoção de medidas para a sua contenção, com reflexos para as práticas de saúde, para as açôes de vigilância epidemiológica, sanitária, e na gestão dos serviços de saúde, ainda não há informaçóes oficiais robustas que subsidiem o conhecimento da situação epidemiológica da coinfecçáo por COVID-19 e TB.

Por ser uma doença determinada socialmente e de baixa visibilidade social, o controle da TB enfrenta desafios incisivos frente à pandemia da COVID-19, podendo comprometer o alcance das metas da Estratégia Global para o Fim da TB, de reduzir em 95\% a mortalidade por essa enfermidade e em $90 \%$ sua incidência, no período de 2015 a 2035. ${ }^{(4)}$ No caso específico do Brasil, também acarretará dificuldades para o cumprimento das recomendaçóes do Plano Nacional pelo Fim da Tuberculose, que estabelece objetivos e atividades específicas pautados nas metas de redução do coeficiente de incidência para menos de dez casos $/ 100.000$ habitantes e do coeficiente de mortalidade por TB para menos de um óbito/ 100.000 habitantes até 2035. ${ }^{(5)}$

A pandemia exacerba as desigualdades em saúde. Em países em desenvolvimento, como no Brasil, a desigualdade social representa terreno fértil para a disseminação da COVID-19, dificultando o cumprimento das recomendaçóes sanitárias, restringindo o acesso aos insumos básicos para higiene e equipamentos de proteção individual, e a assistência ofertada pelos serviços da rede de atenção à saúde. ${ }^{(6)}$

Em se tratando da TB, que está associada às precárias condiçôes de vida, saúde e trabalho, ${ }^{(7)}$ na emergência da COVID-19 se prevê a intensificação dos desafios a serem enfrentados. ${ }^{(8)}$ Os programas de controle da TB podem ser afetados pela necessidade de reorganização dos serviços e profissionais de saúde em função da assistência à COVID-19, além da eventual indisponibilidade de insumos e medicamentos para seu manejo e controle. Ademais, o distanciamento social pode interferir na realização do tratamento diretamente observado (TDO) e no seguimento periódico das pessoas com TB. ${ }^{(2)}$

A OMS manifestou preocupação com a possibilidade de comprometimento do progresso alcançado 
em relação à prevenção e ao tratamento da TB, ressaltando a importância dos programas nacionais da doença garantirem a continuidade das açóes destinadas ao controle da enfermidade durante a pandemia, por meio de abordagens inovadoras centradas nas pessoas ${ }^{1}$. Por sua vez, o Ministério da Saúde brasileiro recomendou aos Programas Estaduais e Municipais de Controle da TB, o uso de tecnologias disponíveis para o seguimento do tratamento da TB, além da dispensação mensal de medicamentos, da priorização do atendimento às pessoas com sintomatologia, assim como a solicitação de exames para $\mathrm{TB}$ aos suspeitos de COVID-19, dentre outras açóes. ${ }^{(9)}$

Reconhecendo que a realidade imposta pela pandemia da COVID-19 afeta sobremaneira o controle da TB no Brasil e no mundo e por se tratar de temática que ainda carece de conhecimento, o presente estudo teve como objetivo: identificar as evidências científicas sobre o impacto da pandemia de COVID-19 na atenção e no controle da TB.

\section{Métodos}

Trata-se de uma Revisão de Escopo, pois é o método mais adequado para detectar características especificas ou conceitos em estudos primários e mapeá-los, tendo como proposito a identificação das evidencias disponíveis sobre um tópico e das lacunas de conhecimento. ${ }^{(10)} \mathrm{O}$ levantamento nas bases de dados foi realizado em junho de 2020. Esta revisão foi registrada na base Open Science Framework. A OMS salienta a importância das revisóes em momentos desafiadores, como em outras pandemias ${ }^{11}$, para a busca de alternativas para a tomada de decisóes em saúde, e responder de modo apropriado a uma questão clínica ou relacionada à política em saúde.

A questáo de pesquisa foi elaborada por meio da estratégia PCC, sendo que os elementos do mnemônico $\mathrm{P}$ (população), C (conceito) e C (contexto) foram: TB e COVID-19, pandemia da COVID-19 e impacto no controle e na atenção à TB, respectivamente. Sendo assim, o presente estudo foi norteado pela seguinte questão: Quais são as evidências científicas que dizem respeito ao impacto da pandemia de COVID-19 no controle e na reorganização da atenção à TB?
Para o estudo, foram considerados como impacto, os efeitos epidemiológicos e clínicos positivos e negativos da pandemia da COVID-19 na atenção à TB.

A busca eletrônica foi conduzida pelos descritores: ("coronavirus"[MeSH Terms] OR "coronavirus"[All Fields])) OR COVID-19[All Fields] OR SARS-CoV-2[All Fields] and tuberculosis. Foram consideradas as seguintes bases de dados gerais: Cochrane Library (via Wiley); Embase (via Elsevier); LILACS; Medical Literature Analysis and Retrieval System Online (MEDLINE), PubMed, Web of Science, Cinahl e Scopus. Também foi realizada busca eletrônica na base de literatura cinzenta: Opengrey (https://opengrey.eu) e busca manual.

O quadro 1 apresenta as estratégias de busca nas diferentes bases de dados.

Quadro 1. Distribuição das estratégias de busca segundo base de dados

\begin{tabular}{|l|}
\hline \multicolumn{1}{|c|}{ Base de dados/ Estratégia de busca } \\
\hline - PUBMED: ("coronavirus"[MeSH Terms] OR "coronavirus"[All Fields]) OR "covid 19"[All \\
Fields] AND tuberculosis[Abstract] = 281 resultados \\
- LILACS: "coronavirus" OR "covid 19" AND "tuberculosis" = 05 resultados \\
- MEDLINE: (covid-19 or coronavirus) AND tuberculosis $=126$ resultados \\
- EMBASE: ('coronavirus infection' OR 'covid 19') AND tuberculosis $=163$ resultados \\
- CINAHL: (covid-19 or coronavirus) AND tuberculosis $=14$ resultados \\
- WEB OF SCIENCE: ("coronavirus" OR "covid 19") AND "tuberculosis" $=97$ resultados \\
- COCHRANE LIBRARY: "coronavirus" in Title Abstract Keyword OR "Covid 19" in Title Abstract \\
Keyword AND "tuberculosis" in Title Abstract Keyword = 07 resultados (Cochrane Reviews) \\
- OPEN GRAY: Opengrey: coronavirus AND tuberculosis $=00$ resultados \\
- SCOPUS: (TITLE-ABS-KEY ("coronavirus" OR "covid 19" ) AND TITLE-ABS-KEY \\
(tuberculosis )) = 278 resultados
\end{tabular}

O processo de seleção dos estudos foi realizado por dois revisores independentes e divergências foram resolvidas por consenso. A seleção dos estudos foi realizada em duas etapas. Na primeira foram avaliados os títulos e resumos das referências identificadas na estratégia de busca e foram pré-selecionados os estudos potencialmente elegíveis. $\mathrm{Na}$ segunda etapa, foi realizada a avaliação na íntegra dos estudos pré-selecionados, para confirmar sua elegibilidade. $\mathrm{O}$ processo de seleção foi realizado por meio da plataforma Rayyan (https://rayyan.qcri.org). ${ }^{(12)}$

\section{Resultados}

Foram identificados, inicialmente, 684 estudos, dos quais, após remoção das duplicações e exclusão, segundo os critérios de inclusão estabelecidos, foram considerados 30 estudos (Figura 1). 


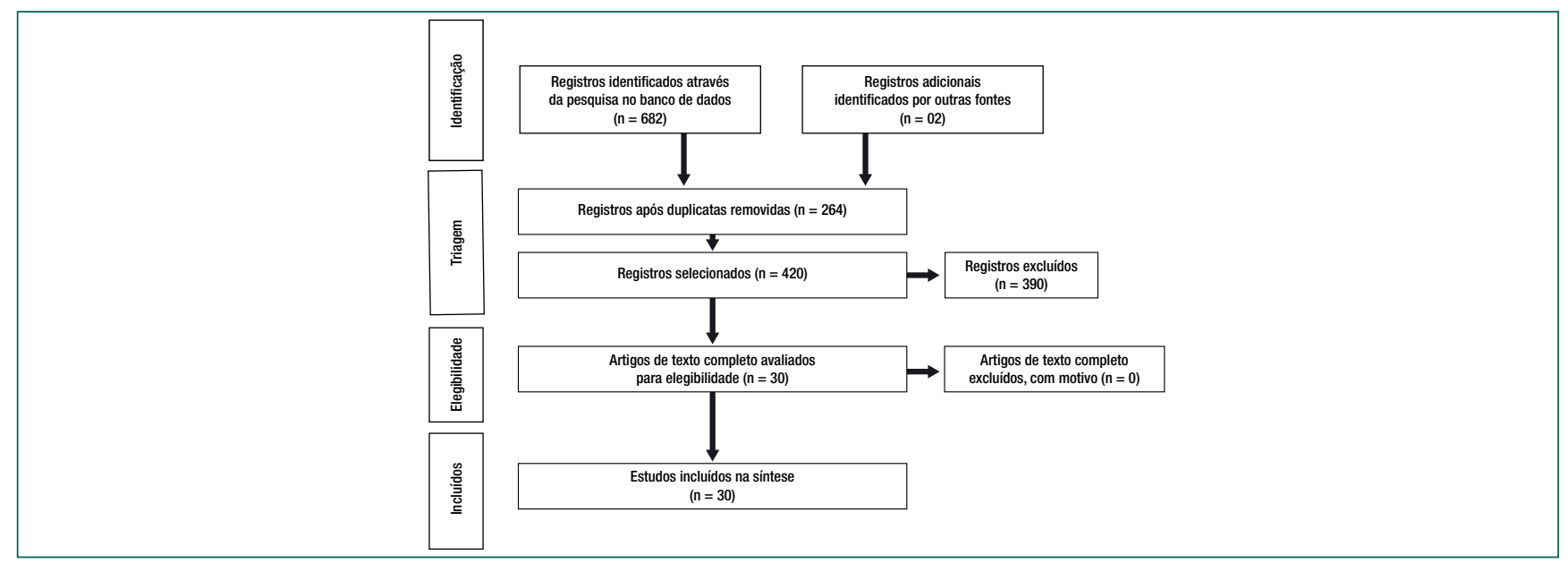

Figura 1. Fluxograma Preferred Reporting Items for PRISMA Extension for Scoping Reviews (PRISMA-SCR) sobre a seleção dos estudos

Em relaçáo ao tipo de publicação, dos 30 estudos analisados, oito consistiam em correspondência, quatro eram revisóes, três eram cartas, dois estudos de caso, editorial e perspectiva, respectivamente, e um tratava de recomendação, notícia, recurso, breve comunicação e relatório, comentário, opinião, nota técnica e modelo de análise, respectivamente. Sobre o local do estudo, em 19 publicações não foi explicitado, três ocorreram na África do Sul e duas publicaçóes envolveram oito países cada. As demais publicaçôes tiveram como cenário o Vietnã, China, Portugal, Canadá, El Salvador e Nigéria.

Os estudos foram categorizados no quadro 2, considerando: Referência, Tipo de publicação, Local do estudo e Resultados.

Quadro 2. Sumário das características dos estudos incluídos

\begin{tabular}{|c|c|c|c|}
\hline Referência & $\begin{array}{l}\text { Tipo de } \\
\text { publicação }\end{array}$ & $\begin{array}{l}\text { Local do } \\
\text { estudo }\end{array}$ & Resultados \\
\hline $\begin{array}{l}\text { World Health Organization. Information Note } 12 \text { May } 2020 . \\
\text { Tuberculosis and COVID-19. World Health Organization; } \\
\text { 2020.(1) }\end{array}$ & Nota técnica & Não se aplica & $\begin{array}{l}\text { Recomenda: fornecimento de estoques adequados de medicamentos para a TB, a fim de } \\
\text { garantir a continuidade do tratamento de forma auto- administrada, para evitar exposição } \\
\text { desnecessária, além de recomendar o uso de tecnologias digitais. }\end{array}$ \\
\hline $\begin{array}{l}\text { Togun T, Kampmann B, Stoker NG, Lipman M. Anticipating } \\
\text { the impact of the COVID-19 pandemic on TB patients and TB } \\
\text { control programmes. Ann Clin Microbiol Antimicrob 2020; } \\
\text { 19(1):21. }\end{array}$ & Revisão & Não se aplica & $\begin{array}{l}\text { A discussão de como a pandemia afetará a TB tomou como exemplo diferentes cenários. No } \\
\text { Reino Unido, o redimensionamento de profissionais para atuarem contra a COVID-19, exige } \\
\text { priorizar ações com foco na doença e não na prevenção. } 0 \text { controle da TB na África sofrerá } \\
\text { impacto nos níveis individual, macroeconômico e no sistema de saúde. }\end{array}$ \\
\hline $\begin{array}{l}\text { Tadolini M, Codecasa LR, García-García Jé-Mía, et al. Active } \\
\text { tuberculosis, sequelae and COVID-19 co-infection: first } \\
\text { cohort of } 49 \text { cases. Eur Respir J. 2020; 56(1):2001398. }\end{array}$ & Carta & 8 países & $\begin{array}{l}\text { Dos } 49 \text { pacientes com COVID-19 e TB, 53\% tiveram TB antes da COVID-19; } 28,5 \% \text { tiveram } \\
\text { COVID-19 primeiro e 18,3\% receberam ambos os diagnósticos no mesmo dia ou semana. A } \\
\text { quase totalidade (exceto um) apresentava comorbidade. A taxa de mortalidade foi de } 12,4 \% \text {. }\end{array}$ \\
\hline $\begin{array}{l}\text { Nguyen TA, Cuong QN, Kim ALT, Huong TN, Nguyen HN, Fox } \\
\text { GJ, Marks GB. Adapting a TB contact investigation strategy } \\
\text { for COVID-19. Int J Tuberc Lung Dis. 2020: 24(5): 548-50. }{ }^{(14)}\end{array}$ & Correspondência & Vietnã & $\begin{array}{l}\text { Realizou-se investigação de contatos para TB adaptando à COVID-19. Foi proposto um } \\
\text { fluxograma para triagem de SARS-CoV-2 e rastreamento em locais com baixo nível de } \\
\text { transmissibilidade comunitária. }\end{array}$ \\
\hline $\begin{array}{l}\text { Alagna R, Besozzi G, Codecasa LR, et al. Celebrating World } \\
\text { Tuberculosis Day at the time of COVID-19. Eur Respir J } \\
\text { 2020; 55: 2000650.15) }\end{array}$ & Carta & Não se aplica & $\begin{array}{l}\text { Destacam-se semelhanças no enfrentamento das duas enfermidades: sobrecarregam o sistema de } \\
\text { saúde, necessidade de diagnóstico rápido e conscientização da população, causam estigma social e } \\
\text { ausência de um sistema de vigilância para o monitoramento de forma compartilhada. }\end{array}$ \\
\hline $\begin{array}{l}\text { CInar OE, Sayinalpb B, Karakulaka EA, Karatasa AA, Veleta M, } \\
\text { Inkayac AC, et al. Convalescent (immune) plasma treatment } \\
\text { in a myelodysplastic COVID-19 patient with disseminated } \\
\text { tuberculosis Transfusion and Apheresis Science 2020. }{ }^{(16)}\end{array}$ & Estudo de caso & Não se aplica & $\begin{array}{l}\text { Apresenta o caso de um paciente com TB, imunocomprometido devido à síndrome } \\
\text { mielodisplásica e com COVID-19, que recebeu tratamento com duas terapias imunológicas de } \\
\text { plasma imune convalescente. }\end{array}$ \\
\hline $\begin{array}{l}\text { Boffa J, Mhlaba T, Sulis G, Moyo S, Sifumba Z, Pai M, et al. } \\
\text { CoVID-19 and tuberculosis in South Africa: A dangerous } \\
\text { combination. S Afr Med J 2020;110(5):341-2.177 }\end{array}$ & Correspondência & África do Sul & $\begin{array}{l}\text { Apresenta preocupação sobre a situação do sistema de saúde e de comunidades carentes } \\
\text { da África do Sul. Profissionais de saúde devem estar atentos para a possibilidade de dupla } \\
\text { infecçã̃o (COVID-19 e TB) em PVHA. }\end{array}$ \\
\hline $\begin{array}{l}\text { Yadav SR, Kumar R, Kumar A, ISH P, Gupta N, Chakrabarti S. } \\
\text { COVID-19: Avoiding a second tragedy in a tuberculosis burdened } \\
\text { country. Monaldi Archives for Chest Disease 2020; 90(2):1338.(18) }\end{array}$ & Carta & Não se aplica & $\begin{array}{l}\text { Medidas já utilizadas no controle da TB como triagem, práticas de etiqueta da tosse, } \\
\text { investigação de contatos e medidas de isolamento podem ser aplicados na prevenção da } \\
\text { COVID-19. }\end{array}$ \\
\hline $\begin{array}{l}\text { He G, Wu J, Shi J, Dai J, Ga,er M, Jiang X, et al. COVID-19 in } \\
\text { Tuberculosis Patients: A Report of Three Cases. J Med Virol } \\
\text { 2020;10.199) }\end{array}$ & Estudo de caso & China & $\begin{array}{l}\text { Relato de três pacientes com TB pulmonar e COVID-19, que foram seguidos prospectivamente } \\
\text { desde a internação até a alta. Dois idosos evoluíram para formas graves da COVID-19 e tiveram } \\
\text { um longo processo de recuperação. }\end{array}$ \\
\hline $\begin{array}{l}\text { Saunders MJ, Evans CA. COVID-19, tuberculosis, and } \\
\text { poverty: preventing a perfect storm. Eur Respir J 2020;56: } \\
\text { 2001348. }{ }^{(20)}\end{array}$ & Editorial & Não se aplica & $\begin{array}{l}\text { Com o aumento de pessoas vivendo em situação de pobreza, ocorrerá um aumento de casos } \\
\text { de TB, redução de recursos humanos e materiais para o seu controle, além de impacto no } \\
\text { acompanhamento do tratamento e na investigaçãa de suspeitos, na provisão de medicamentos, } \\
\text { aumento do estigma e comprometimento de medidas de proteção social. }\end{array}$ \\
\hline
\end{tabular}


Continuação.

\begin{tabular}{|c|c|c|c|}
\hline Referência & $\begin{array}{l}\text { Tipo de } \\
\text { publicação }\end{array}$ & $\begin{array}{l}\text { Local do } \\
\text { estudo }\end{array}$ & Resultados \\
\hline $\begin{array}{l}\text { Cox V, Wilkinson L, Grimsrud A, Hughes J, Reuter A, Conrade F. } \\
\text { Critical changes to services for TB patients during the COVID-19 } \\
\text { pandemic. Int J Tuberc Lung Dis 2020;24(5):542-4. }{ }^{21)}\end{array}$ & Correspondência & Não se aplica & $\begin{array}{l}\text { Descreve mudanças decorrentes da pandemia para proteger as pessoas com TB da exposição ao } \\
\text { SARs-COv-2, como: diminuição de idas desnecessárias aos serviços de saúde, atendimento prioritário e } \\
\text { monitoramento por telefone para garantir a adesão ao tratamento da TB. }\end{array}$ \\
\hline $\begin{array}{l}\text { Peixoto VR, Mexia R, Santos NS, Carvalho C, Abrantes A. From } \\
\text { Tuberculosis to COVID-19: Legal and Constitutional Framework } \\
\text { Regarding Compulsory Isolation/reatment due to Contagious } \\
\text { Diseases in Portugal. Acta Med Port 2020;33(4):225-8. }\end{array}$ & Perspectiva & Portugal & $\begin{array}{l}\text { Aponta-se que devem ser analisadas, para a tomada de decisões, a proporcionalidade entre } \\
\text { riscos e medidas de saúde pública, e o equilíbrio entre os direitos, liberdades e garantias dos } \\
\text { cidadãos, além da defesa da Saúde Pública. }\end{array}$ \\
\hline $\begin{array}{l}\text { Pang Y, Lin Y, Du J, Gao J, Li L. Impact of COVID-19 } \\
\text { on tuberculosis control in China. Int J Tuberc Lung Dis } \\
\text { 2020;24(5):545-7.(23) }\end{array}$ & Correspondência & Não se aplica & $\begin{array}{l}\text { As mudanças na organização dos serviços de saúde por conta da COVID-19 podem } \\
\text { comprometer o diagnóstico precoce, considerando o receio em buscar atendimento, bem como } \\
\text { a interrupção do tratamento da TB. }\end{array}$ \\
\hline $\begin{array}{l}\text { Kiddell-Monroe R, Ranta M, Enook S, Saranchuk P. Inuit } \\
\text { communities can beat COVID-19 and tuberculosis. The } \\
\text { Lancet 2020; } 5 .^{\left({ }^{24)}\right.}\end{array}$ & Correspondência & Canadá & $\begin{array}{l}0 \text { vírus pode atingir a população de Nunavut (comunidade de Inuítes) e levar a consequências } \\
\text { trágicas, pois o acesso ocorre apenas por via área, as instalações médicas são básicas e não } \\
\text { há testes suficientes para a COVID-19. }\end{array}$ \\
\hline $\begin{array}{l}\text { El Salvador. Ministerio de Salud. Lineamientos Técnicos para } \\
\text { el Abordaje y Seguimiento de casos de Tuberculosis, ante la } \\
\text { Emergencia Nacional por Covid-19. El Salvador; 2020. }{ }^{25)}\end{array}$ & Recomendação & El Salvador & $\begin{array}{l}\text { O documento técnico apresenta: modalidades de tratamento (supervisionado no serviço } \\
\text { de saúde, no domicilio, por vídeo-chamada), ações para a prevenção da COVID-19 em } \\
\text { pessoas com TB; ações dos profissionais de saúde para a detecção de novos casos de TB e } \\
\text { biossegurança na rede de laboratórios. }\end{array}$ \\
\hline $\begin{array}{l}\text { Dara M, Sotgiu G, Reichler MR, Chiang CY, Chee CBE, Migliori } \\
\text { GB. New diseases and old threats: lessons from tuberculosis } \\
\text { for the COVID-19 response. Int J Tuberc Lung Dis 2020; } \\
\text { 24(5):544-5. }{ }^{(26)}\end{array}$ & Correspondência & Não se aplica & $\begin{array}{l}\text { Ações para o controle da TB podem ser complementares ao controle da COVID-19, como: } \\
\text { capacitação conjunta com setores de vigilância epidemiológica, uso de ferramentas } \\
\text { diagnósticas e práticas desenvolvidas no controle da TB, como triagem, etiqueta respiratória, } \\
\text { investigação dos contatos, controle de infecção e o distanciamento social. }\end{array}$ \\
\hline $\begin{array}{l}\text { Riccò M, Gualerzi G, Ranzieri S, Bragazzi NL. Stop playing } \\
\text { with data: there is no sound evidence that Bacille Calmette- } \\
\text { Guérin may avoid SARS-CoV-2 infection (for now). Acta } \\
\text { Biomed 2020;91(2):207-13. }{ }^{(27}\end{array}$ & Revisão & Não se aplica & $\begin{array}{l}\text { Identificaram-se pesquisas em andamento sobre como a política de vacinação da BCG pode ter } \\
\text { afetado a pandemia, em termos de incidência e/ou mortalidade. }\end{array}$ \\
\hline $\begin{array}{l}\text { Nordling L. Tested by HIV and TB, South Africa confronts new } \\
\text { pandemic. Science 2020; 368(6487):117. }{ }^{(28)}\end{array}$ & Notícia & África do Sul & $\begin{array}{l}\text { Clínicas móveis de teste para COVID-19 foram enviadas para áreas densamente povoadas. } \\
\text { Institutos que realizavam pesquisas sobre TB e HIV redirecionaram suas equipes; uma das } \\
\text { pesquisas investiga o uso da cloroquina e, outras, a possibilidade de diagnosticar casos } \\
\text { assintomáticos de COVID-19 e a associação TB/HIV. }\end{array}$ \\
\hline $\begin{array}{l}\text { Manyazewal T, Woldeamanuel Y, Fekadu B, Marconi VC. } \\
\text { The fight to end tuberculosis must not be forgotten in the } \\
\text { COVID-19 outbreak. Nature MEd 2020;26(6):811-2.(29) }\end{array}$ & Correspondência & Não se aplica & $\begin{array}{l}\text { Durante a pandemia espera-se um aumento de casos de TB e desenvolvimento de resistência } \\
\text { aos medicamentos. Governos devem buscar estratégias para garantir a adesão ao tratamento } \\
\text { da TB. }\end{array}$ \\
\hline $\begin{array}{l}\text { Adepoju P. Tuberculosis and HIV responses threatened by } \\
\text { COVID-19. The Lancet 2020; 7(5): e319-20. }{ }^{(30)}\end{array}$ & Recurso & Nigéria & $\begin{array}{l}\text { A COVID-19 vem afetando medidas de controle da TB e HIV. } 0 \text { fornecimento e o transporte } \\
\text { dos medicamentos para TB têm risco de serem interrompidos. A escassez de equipamentos } \\
\text { de proteção, a falta de treinamento dos profissionais de saúde, em relação ao diagnóstico } \\
\text { diferencial entre TB e COVID-19 e a dificuldade de deslocamento aos serviços de saúde, podem } \\
\text { contribuir para a piora da situação de saúde. }\end{array}$ \\
\hline $\begin{array}{l}\text { Yasri S, Wiwanitkit V. Tuberculosis and novel Wuhan coronavirus } \\
\text { infection: Pathological interrelationship. Indian J Tuberc 2020. }{ }^{(1)}\end{array}$ & Correspondência & Não se aplica & $\begin{array}{l}\text { Dados de } 21 \text { de janeiro de 2020, apresentaram } 221 \text { casos de nova infecção pelo novo } \\
\text { coronavírus, sendo } 1 \text { caso de coinfecção com TB }(0,45 \%) \text { e } 6 \text { óbitos. }\end{array}$ \\
\hline $\begin{array}{l}\text { Motta RC, DÁmbrosio L, García-García JM, Golettu D, } \\
\text { Gualano G, Lipani F. Tuberculosis, COVID-19 and migrants: } \\
\text { Preliminary analysis of deaths occuring in } 69 \text { patients from } \\
\text { two cohorts. Pulmonol 2020;26(4):233-40. }\end{array}$ & $\begin{array}{l}\text { Breve } \\
\text { comunicação }\end{array}$ & 8 países & $\begin{array}{l}\text { De } 69 \text { pacientes observou-se oito óbitos, sendo a maioria }(n=7) \text { do sexo masculino e idade } \\
\text { média de } 70 \text { anos. } 0 \text { diagnóstico da TB foi anterior ao da COVID-19, sendo que apenas um } \\
\text { deles teve diagnóstico simultâneo de COVID-19 e TB; os demais tiveram diagnóstico de } \\
\text { COVID-19 entre } 7 \text { e } 75 \text { dias (mediana de 22,5 dias) após o diagnóstico de TB. }\end{array}$ \\
\hline $\begin{array}{l}\text { Sharkie IK. BCG is a Good Immunotherapeutic Agent for Viral and } \\
\text { Autoimmune Diseases: Is it a New Weapon against Coronavirus } \\
\text { (COVID-19)? Electron J Gen Med. 2020;17(6):em229.'33) }\end{array}$ & Revisão & Não se aplica & $\begin{array}{l}\text { A ação desencadeada pela vacina BCG no sistema imunológico pode constituir resistência a } \\
\text { uma variedade de doenças e patógenos não relacionados, impactando na redução de alguns } \\
\text { tipos de infecções virais, sendo uma delas a COVID-19. }\end{array}$ \\
\hline $\begin{array}{l}\text { Rajarshi K, Chatterjee A, Ray S. BCG vaccination strategy } \\
\text { implemented to reduce the impact of COVID-19: Hype or } \\
\text { Hope?. Med in Drug Discovery 2020;7. } .^{(34)}\end{array}$ & Revisão & Não se aplica & $\begin{array}{l}\text { Relata-se que se encontram em andamento ensaios clínicos que envolvem a vacina BCG em } \\
\text { profissionais de saúde que atuam na linha de frente da COVID-19, indagando-se se a vacina } \\
\text { reduz a gravidade e ocorrência da doença. }\end{array}$ \\
\hline $\begin{array}{l}\text { Redelman-Sidi G. Could BCG be used to protect against } \\
\text { COVID-19? Nature Rev Urol 2020;17(6):316-7.(35) }\end{array}$ & Comentário & Não se aplica & $\begin{array}{l}\text { Pesquisas estão sendo desenvolvidas para testar a hipótese de que a vacina BCG protege contra } \\
\text { a COVID-19. Caso os resultados comprovem efeito protetor, apontam-se as indagaçôes: Quanto } \\
\text { tempo dura a imunidade após a vacinação? Qual o momento ideal para vacinar? }\end{array}$ \\
\hline $\begin{array}{l}\text { Covián C, Retamal-Díaz A, Sueno SM, Kalergis AM. Could } \\
\text { BCG Vaccination Induce Protective Trained Immunity for } \\
\text { SARS-CoV-2? Front. Immunol 2020;11. }{ }^{(36)}\end{array}$ & Perspectiva & Não se aplica & $\begin{array}{l}\text { Países onde a vacinação com BCG é administrada ao nascer mostraram menor taxa de } \\
\text { contágio e mortes relacionadas à COVID-19, sugerindo que esta vacina pode induzir imunidade } \\
\text { treinada e conferir alguma proteção à COVID-19. }\end{array}$ \\
\hline $\begin{array}{l}\text { Hergarty PKm Sfakianos JP, Giannarini G, Dinardo AR, Kamat } \\
\text { AM. COVID-19 and Bacillus Calmette-Gueri: What is the } \\
\text { Link? Eur Uro Oncol 2020;3(3): 259-61. }\end{array}$ & Editorial & Não se aplica & $\begin{array}{l}\text { Observou-se que países da Europa mais afetados pela COVID-19 foram aqueles que não } \\
\text { contavam com programa nacional de vacinação da BCG; a incidência em países com vacinação } \\
\text { foi de } 0,8 \text { por milhão e } 34,8 \text { por milhão naqueles sem esse programa. }\end{array}$ \\
\hline $\begin{array}{l}\text { Maciel EN, Gonçalves-Júnior E, Dalcolmo MMP. Tuberculosis } \\
\text { and coronavirus: what do we know? Epidemiol Serv Saude } \\
\text { 2020; 29(2):e2020128. }{ }^{(38)}\end{array}$ & Opinião & Não se aplica & $\begin{array}{l}\text { A incidência elevada da TB, em alguns estados brasileiros, acrescida da alta densidade } \\
\text { populacional observada nas periferias das grandes cidades, traara obstáculos para o } \\
\text { enfrentamento da COVID-19. }\end{array}$ \\
\hline $\begin{array}{l}\text { Amimo F, Lambert B, Magit A. What does the COVID-19 } \\
\text { pandemic mean for HIV, tuberculosis, and malaria control? } \\
\text { Trop Med Health 2020; 48:32. }{ }^{\left({ }^{9}\right)}\end{array}$ & Breve relatório & África do Sul & $\begin{array}{l}0 \text { estudo avaliou o impacto da COVID-19 no controle do HIV, TB e malária na África. As medidas } \\
\text { para o controle da COVID-19 negligenciam a realidade epidemiológica, social e econômica. As } \\
\text { medidas de isolamento e limitações de alguns setores como viagens e negócios, determinam } \\
\text { que a população em idade produtiva enfrente dificuldades para acessar serviços essenciais. }\end{array}$ \\
\hline $\begin{array}{l}\text { Stop TB Partnership. The potential impact of the COVID-19 } \\
\text { response on tuberculosis in high-burden countries: a } \\
\text { modelling analysis [Internet]. Stop TB Partnership, Geneva: } \\
\text { Stop TB Partnership, Geneva; } 2020 \text { Stop TB Partnership. }{ }^{(40)}\end{array}$ & Modelo de análise & Não se aplica & $\begin{array}{l}\text { A análise de modelagem para estimar o impacto da COVID- } 19 \text { em três países com alta carga } \\
\text { de TB (Índia, República do Quênia e Ucrânia), sugere redução de } 25 \% \text { na detecção da TB e } \\
\text { aumento de } 13 \% \text { na mortalidade por TB. Espera-se, entre } 2020 \text { e 2025, 6,3 milhões de casos } \\
\text { adicionais de TB. }\end{array}$ \\
\hline
\end{tabular}


A leitura e análise dos artigos resultou nas seguintes categorias de análise e seus elementos constitutivos (Quadro 3).

Quadro 3. Categorias de análise e seus elementos constitutivos

\begin{tabular}{|l|l|l|}
\hline $\begin{array}{l}\text { Aumento da } \\
\text { vacina BCG na } \\
\text { COVID }\end{array}$ & $\begin{array}{l}\text { Impact3-37) } \\
\text { COVID-19 no controle da } \\
\text { TB }^{(2,13,14,17,19-22,24,29-32,38-39,41)}\end{array}$ & $\begin{array}{l}\text { Efeitos da COVID-19 na } \\
\text { organização dos serviços de }_{\text {saúde }^{(1,15,16,18,23,25-26,28)}}\end{array}$ \\
\hline - Efeitos da Vacina & - Influência da COVID-19 no & - Necessidade de reorganização \\
BCG na COVID-19 & seguimento e na adesão ao & de recursos humanos e materiais \\
& tratamento da TB & voltados à TB e outros agravos \\
para o enfrentamento da COVID-19 \\
& - COVID-19 agrava o contexto \\
& de vulnerabilidade social com \\
& implicações no controle da TB & àdanças gerais na assistência \\
& & \\
\hline
\end{tabular}

\section{Discussão}

Os estudos incluídos nesta revisão mostraram a amplitude do problema da TB e o impacto na pandemia. A maior parte dos temas dos artigos apontam o impacto da COVID-19 no controle da TB, principalmente no que se refere aos problemas e dificuldades que a pandemia acarretará, a exemplo das necessárias medidas de distanciamento social, e suas repercussóes, como acesso à detecção precoce, seguimento e adesão ao tratamento da TB. Nessa linha, ainda se destaca a deterioração das condições de vida e de trabalho, com aumento importante na vulnerabilidade social. Quanto aos efeitos da COVID-19 nos sistemas de saúde aponta-se, principalmente, a necessária reorganização dos serviços para atender as demandas da pandemia, com implicações na assistência aos pacientes com TB. Uma outra categoria importante de análise, refere-se às implicações da vacinação BCG, principalmente em relação às indagaçôes sobre o seu efeito protetor na evolução da COVID-19.

Esta revisão buscou sintetizar o estado da arte sobre a TB e a COVID-19, com base nas categorias apresentadas. A categoria Impacto da pandemia da COVID-19 no controle da TB congrega a maior parte das referências identificadas na literatura, evidenciando-se preocupação dos pesquisadores, em especial, porque a pandemia pode comprometer conquistas alcançadas no controle da TB ao longo de décadas, constituindo obstáculo para o alcance das metas estabelecidas pela Estratégia Fim da TB.
A COVID-19 ultrapassou a TB no ranking de doenças infecciosas que mais matam no mundo. É indubitável que a pandemia aprofunda as desigualdades sociais, estendendo-se para além da crise na saúde. Essa crise ameaça afetar de forma desproporcional as populaçóes de países menos desenvolvidos, da África, Sudeste Asiático e América Central e do Sul. Em consequência, dada a consagrada determinação social da TB, aponta-se a probabilidade do aumento dos casos nos países que compóem esse grupo e que apresentem alta densidade populacional. ${ }^{(20-38)}$

Quanto à categoria Efeitos da COVID-19 na organização dos serviços de saúde, é possível observar semelhanças entre a TB e a COVID-19. As semelhanças relacionam-se: à importância do diagnóstico precoce e da conscientização para o controle de doenças infecciosas, ao estigma social que ambas as enfermidades carreiam, à falta de conhecimento a respeito da suscetibilidade de alguns grupos à infecção e à importância de uma plataforma para o compartilhamento dos dados. Por outro lado, assinala-se a discrepância de investimentos para o controle da COVID-19, assim como a agilidade na reorganização de sistemas de saúde, por parte de alguns países, substantivamente no âmbito da atenção hospitalar, principalmente disponibilizando-se leitos e equipamentos de terapia intensiva. ${ }^{(15)}$ Destaca-se aqui a necessária velocidade na adaptação dos sistemas de saúde frente à emergência pandêmica. Principalmente no Brasil sublinha-se que a pandemia da COVID-19 determinou a injeção de recursos financeiros, sobretudo no âmbito hospitalar e de alta complexidade, e principalmente nas unidades de saúde de caráter privado, deixando de lado a necessária atenção à vigilância de casos no território, no âmbito público da Atenção Primária em Saúde.

A pandemia trouxe desafios adicionais para diversos setores, para além da vigilância epidemiológica, na esfera nacional e internacional, mas também incidindo nas relaçôes internacionais e no necessário reconhecimento da necessidade de políticas públicas voltadas à redução das desigualdades de acesso aos sistemas de saúde, e da urgente redução da injustiça social. Aliada à fundamental reversão desse quadro destaca-se a importância de algumas estratégias para 
a contenção da pandemia, tais como: disponibilidade de testes em larga escala, logística de saúde e áreas afins para o isolamento territorial e monitoramento dos casos positivos e contatos, investimentos para a proteção de profissionais de saúde, provisão de equipamentos e insumos médicos apropriados e de reconhecida eficácia, assim como as necessárias políticas, por parte do Estado, para a manutenção da proteção social da população, e de estratégias para a retomada programada e segura, com a manutenção de açóes e serviços prioritários, especialmente dos programas essenciais para a saúde como o Programa de Controle da TB. ${ }^{(40,41)}$

A pandemia também causa impacto na organização dos serviços de saúde, com destaque na redução de recursos humanos e materiais destinados ao controle da TB, ${ }^{(20)}$ além da necessária reorganização dos serviços antes destinados à $\mathrm{TB}$ para o atendimento de casos de COVID-19, ${ }^{(23)}$ escassez de materiais de proteção, falta de treinamento dos profissionais de saúde em relação ao diagnóstico diferencial de TB e COVID-19, assim como em relação às precauçóes de segurança, uso adequado de equipamentos de proteção individual e com referência aos critérios de isolamento. $^{(21,30)}$

No âmbito dos serviços ambulatoriais, o manejo de recursos humanos para atuar na linha de frente contra a COVID-19 repercute na operacionalização do TDO e nas açôes de busca ativa de sintomáticos respiratórios, estas últimas também comprometidas pelo necessário distanciamento social, atreladas às barreiras de acesso aos serviços de saúde. Todos esses aspectos podem refletir de forma negativa no diagnóstico precoce dos casos de TB, nos indicadores da doença e no aumento de cepas resistentes aos medicamentos, ${ }^{(29,42)}$ assim como nos processos de adesão ao tratamento e no monitoramento dos casos e de seus comunicantes. ${ }^{(2)} \mathrm{Um}$ outro aspecto relevante, e que tem impacto no diagnóstico precoce da TB, é o estigma, materializado pela presença da tosse, situação em que o usuário pode hesitar em procurar o serviço de saúde. ${ }^{(2)}$

O distanciamento social é recomendado como medida fundamental para a contenção do avanço da COVID-19. ${ }^{(14,22,30)}$ No entanto, a restrição de comparecimentos ao serviço de saúde pode comprome- ter a adesão ao tratamento da TB e levar a desfechos desfavoráveis. Somado às limitaçóes econômicas, provocadas pela pandemia, estas influenciam negativamente no acesso aos serviços essenciais, também devido aos custos médicos e à falta de transporte para chegar às unidades de saúde. ${ }^{(39)}$

Com vistas à superação desses impactos, as publicações sugerem a diminuição de idas desnecessárias aos serviços de saúde, o monitoramento via contato telefônico ou por meio de plataformas digitais, além da priorização do atendimento quando presencial. ${ }^{(21,23)}$ Também sugerem o fornecimento de estoques adequados de medicamentos para a conclusão do tratamento da TB de forma auto-administrada ${ }^{1}$, a rápida restauração dos serviços de $\mathrm{TB}$ e a intensificação da busca ativa de casos, incluindo a investigação de contatos por meio de tecnologias digitais. $^{(42)}$

Um estudo que buscou analisar duas realidades distintas, no Reino Unido e na África, demonstrou o impacto negativo da pandemia da COVID-19 em relação às ações de $\mathrm{TB}$, visto que foi necessário priorizar ações voltadas para a primeira e não para a prevenção da TB. Além disto, destacou que a diminuiçãa da circulação das pessoas com TB nos serviços de saúde pode comprometer o vínculo estabelecido e, consequentemente, a adesão ao tratamento. Problematizar aspectos da interação entre ambas as enfermidades possibilita o fundamental aprendizado entre comunidades, profissionais de saúde e formuladores de políticas, assinalando-se o necessário reconhecimento da relação entre muitas doenças infecciosas e as iniquidades sociais ${ }^{2}$. Por outro lado, além do reconhecimento dessa associação, requer-se o reconhecimento das necessárias mudanças estruturais nas sociedades, de forma a conduzir os grupos sociais à igualdade no acesso aos bens que proporcionam a dignidade para a condução da vida.

No que diz respeito à categoria Efeito da vacina BCG na COVID, ressalta-se a importância de os profissionais de saúde estarem atentos para a ocorrência de ambas as enfermidades concomitantemente. À medida que a pandemia avança, mais pessoas com TB terão sido expostas ao Sars-CoV-2 e um resultado positivo para este não exclui a possibilidade de $\mathrm{TB}$, principalmente em ambientes com alta carga. ${ }^{(3)}$ 
$\mathrm{Na}$ presente revisão foram identificadas algumas referências que tratam da utilização da vacina Bacillus Calmette-Guérin (BCG) no enfrentamento da COVID-19, verificando-se consenso sobre a ausência de evidências do efeito protetor da vacina BCG contra infecçôes causadas pelo SARS-CoV-2, e ainda se aguardam os resultados de pesquisas em andamento. ${ }^{(27-33-37)}$ De qualquer forma, estudos clínicos preliminares apontam que a vacina BCG pode induzir uma resposta imune adaptativa no organismo e desencadear proteção contra algumas infecçôes virais, incluindo a COVID-19. Entretanto, uma revisão sistemática sobre a temática questiona a qualidade dos estudos desenvolvidos, principalmente em relação à falta de clareza sobre os fatores de confusão (informação demográfica dos países avaliados, parcela de pessoas residentes em ambientes urbanos, entre outros), e pelo fato de simplesmente terem sido avaliadas diferenças entre incidência e mortalidade da COVID-19 em relação às taxas de vacinação. ${ }^{(27)}$

Ao refletir sobre o cenário epidemiológico da COVID-19 e as experiências no controle da pandemia, são considerados seus potenciais reflexos. Ainda que a rápida evolução tecnológica tenha possibilitado o desenvolvimento de vacinas que possibilitem pelo menos a redução da evolução dos casos para graves e letais, a inexistência de uma vacina específica para evitar a doença, ainda exige ações de distanciamento social, a fim de reduzir a velocidade da curva epidêmica, bem como políticas públicas voltadas para a proteção do trabalhador e investimento no setor saúde. ${ }^{(40)}$

$\mathrm{Na}$ esfera da TB, a OMS reforça que a identificação e o tratamento de pessoas com a doença são pilares fundamentais para a sua prevenção e controle e que estes devem ser mantidos durante a pandemia de COVID-19. Assinala, assim, que os esforços e as açôes de controle e eliminação da TB, tais como prevenção e controle de infecçóes, rastreamento de contatos, cuidados domésticos e comunitários, e sistemas de vigilância e monitoramento, que vem sendo adotados ao longo dos anos, podem ajudar na resposta à COVID-19. ${ }^{(3)}$

Os programas de controle da TB, assim como o princípio fundante do Sistema Único de Saúde, que é a universalidade sofrem com a diminuição de recursos, em decorrência da aprovação da Emenda Constitucional95/2016, que congela os gastos da União com despesas primárias por 20 anos, e quebra o núcleo essencial do direito que é a garantia de recursos orçamentários para a sua sustentabilidade. Nesse sentido, repercute também na priorização de ações para mitigar a COVID-19, na necessária cobertura vacinal e na detecção precoce de casos e nas medidas que podem conter a sua dispersão, assim como na negligência da esfera federal em relação às necessárias medidas de proteção social, com decorrências no cotidiano dos grupos sociais fragilizados pela inserção social.

O histórico de conquistas na implementação de políticas para o controle da TB está ameaçado pela pandemia da COVID-19. Soma-se a isso, ainda, a relação entre a TB e a desigualdade social, sendo esta, também, um dos efeitos da expansão do novo coronavírus, o que ressalta a necessidade de investimentos e mudanças estruturais por parte dos governos nacionais, locais e internacionais. Destacase, também, a importância da sustentabilidade ética e política dos programas de controle da TB, no sentido de manter vigentes as açóes de manejo e vigilância da doença.

\section{Conclusão}

Os estudos analisados permitem concluir que, no período analisado, a literatura científica apresentou, principalmente três categorias de temas relacionados ao impacto da pandemia de COVID-19 na atenção à TB: influência da doença no seguimento e na adesão ao tratamento da TB, verificando-se aspectos que dificultaram tais necessidades, inclusive descontinuidade, principalmente em decorrência do necessário distanciamento social no início da pandemia. A COVID-19 intensificou a vulnerabilidade social, com repercussóes na incidência da TB, principalmente considerando-se que esta última é consagradamente reconhecida como socialmente determinada. O segundo conjunto temático refere-se à necessária organização dos serviços de saúde, atendendo as demandas da COVID-19, com re- 
sultados negativos no controle da TB, em termos de organização dos processos de trabalho e logística de atendimento. $\mathrm{O}$ terceiro conjunto refere-se aos efeitos da vacina BCG sobre a infecção pela SarsCoV-2, verificando-se resultados ainda inconclusivos a respeito. Enfatiza-se preocupação em relação ao impacto da pandemia da COVID-19 na sustentabilidade programática de doenças transmissíveis de caráter crônico, como a TB, que ainda representa um problema mundial de saúde pública e requer atenção prolongada e continuada pelos serviços de saúde.

\section{Referências}

1. World Health Organization (WHO). Coronavirus disease 2019 (COVID-19): situation reports. Genève: WHO; 2020. [cited May 26]. Available from: https://www.who.int/emergencies/diseases/novelcoronavirus-2019/situation-reports

2. Togun T, Kampmann B, Stoker NG, Lipman M. Anticipating the impact of the COVID-19 pandemic on TB patients and TB control programmes. Ann Clin Microbiol Antimicrob. 2020;19(1):21.

3. World Health Organization (WHO). Information Note 12 May 2020. Tuberculosis and COVID-19. Genève:WHO; 2020. [cited May 26]. Available from: https://www.who.int/docs/default-source/documents/ tuberculosis/infonote-tb-covid-19.pdf

4. World Health Organization (WHO). The End TB Strategy. 2015. Genève: WHO; 2015. [cited 2021 May 26]. Available from: https://www.who. int/tb/End_TB_brochure.pdf?ua =1

5. Brasil. Ministério da Saúde. Secretaria de Vigilância em Saúde. Departamento de Vigilância das Doenças Transmissíveis. Brasil Livre da Tuberculose: Plano Nacional pelo Fim da Tuberculose como Problema de Saúde Publica. Brasília (DF): Ministério da Saúde; 2017. [citado 2021 Maio 26]. Disponível em:http://bvsms.saude.gov.br/bvs/ publicacoes/brasil_livre_tuberculose_plano_nacional.pdf

6. Minayo MC, Freire NP. Pandemic exacerbates health inequalities. Cienc Saude Colet.2020;25(9). https://doi.org/10.1590/141381232020259.13742020

7. Bertolozzi MR, Takahashi RF, Franca FO, Hino P. The incidence of tuberculosis and its relation to social inequalities: Integrative Review Study on PubMed Base. Esc Anna Nery. 2020;24(1):e20180367.

8. Oliveira WK, Duarte E, Franca GV, Garcia LP. How Brazil can hold back COVID-19. Epidemiol Serv Saude. 2020;29(2):e2020044.

9. Brasil. Ministério da Saúde. Secretaria de Vigilância em Saúde. Departamento de Doenças de Condições Crônicas e Infecções Sexualmente Transmissíveis. Oficio Circular No 5/2020/CGDR/. DCCI/SVS/MS - Orientações sobre as ações de manejo e controle da tuberculose durante a epidemia do COVID-19. Brasília (DF): Ministério da Saúde; 2020.

10. Munn Z, Peters MD, Stern C, Tufanaru C, McArthur A, Aromataris E. Systematic review or scoping review? Guidance for authors when choosing between a systematic or scoping review approach. BMC Med Res Methodol. 2018 ;18(1):143.
11. Schunemann HJ, Moja L. Reviews: Rapid! Rapid! Rapid! ...and systematic. Syst Rev. 2015 Jan; 4(1):4

12. Ouzzani M, Hammady H, Fedorowicz Z, Elmagarmid A. Rayyan-a web and mobile app for systematic reviews. Syst Rev. 2016;5(1):210.

13. Tadolini M, Codecasa LR, Garcia-Garcia JM, Blanc FX, Borisov S, Alffenaar JW, et al. Active tuberculosis, sequelae and COVID-19 coinfection: first cohort of 49 cases. Eur Respir J. $2020 ; 56(1): 2001398$.

14. Nguyen TA, Cuong QN, Kim AL, Huong TN, Nguyen HN, Fox GJ, et al. Adapting a TB contact investigation strategy for COVID-19. Int J Tuberc Lung Dis. 2020;24(5):548-50.

15. Alagna R, Besozzi G, Codecasa LR. GOri A, Migliori GB, Raviglione M, et al. Celebrating World Tuberculosis Day at the time of COVID-19. Eur Respir J. 2020; 55:2000650.

16. Çınar OE, Sayınalp B, Aladağ Karakulak E, Avşar Karataş A, Velet M, İnkaya $\mathrm{AC}$, et al. Convalescent (immune) plasma treatment in a myelodysplastic COVID-19 patient with disseminated tuberculosis. Transfus Apher Sci. 2020;59(5):102821.

17. Boffa J, Mhlaba T, Sulis G, Moyo S, Sifumba Z, Pai M, et al. COVID-19 and tuberculosis in South Africa: A dangerous combination. S Afr Med J. 2020;110(5):341-2.

18. Yadav SR, Kumar R, Kumar A. ISH P, Gupta N, Chakrabarti S. COVID-19: avoiding a second tragedy in a tuberculosis burdened country. Monaldi Arch Chest Dis. 2020;90(2):1338.

19. He G, Wu J, Shi J, Dai J, Gamber M, Jiang X, et al. COVID-19 in tuberculosis patients: A report of three cases. J Med Virol. 2020 ;92(10):1802-6.

20. Saunders MJ, Evans CA. COVID-19, tuberculosis and poverty: preventing a perfect storm. Eur Respir J. 2020;56(1):2001348.

21. Cox V, Wilkinson L, Grimsrud A, Hughes J, Reuter A, Conradie F, et al. Critical changes to services for TB patients during the COVID-19 pandemic. Int J Tuberc Lung Dis. 2020 ;24(5):542-4.

22. Peixoto VR, Mexia R, Santos NS, Carvalho C, Abrantes A. [From Tuberculosis to COVID-19: Legal and Constitutional Framework Regarding Compulsory Isolation/Treatment due to Contagious Diseases in Portugal]. Acta Med Port. 2020 ;33(4):225-8.

23. Pang Y, Liu Y, Du J, Gao J, Li L. Impact of COVID-19 on tuberculosis control in China. Int J Tuberc Lung Dis. 2020;24(5):545-7.

24. Kiddell-Monroe R, Ranta M, Enook S, Saranchuk P. Inuit communities can beat COVID-19 and tuberculosis. Lancet Public Health. 2020;5(6):e312.

25. El Salvador. Ministerio de Salud. Lineamientos Técnicos para el Abordaje y Seguimiento de casos de Tuberculosis, ante la Emergencia Nacional por Covid-19. El Salvador; 2020. [cited 2021 May 26]. Available from: http://asp.salud.gob.sv/regulacion/pdf/lineamientos/ lineamientostecnicosparaelabordajeyseguimientodecasosde tuberculosisantelaemergencianacionalporCOVID19.pdf

26. Dara M, Sotgiu G, Reichler MR, Chiang CY, Chee CB, Migliori GB. New diseases and old threats: lessons from tuberculosis for the COVID-19 response. Int J Tuberc Lung Dis. 2020;24(5):544-5.

27. Riccò M, Gualerzi G, Ranzieri S, Bragazzi NL. Stop playing with data: there is no sound evidence that Bacille Calmette-Guérin may avoid SARS-CoV-2 infection (for now). Acta Biomed. 2020;91(2):207-13.

28. Nordling L. Tested by HIV and TB, South Africa confronts new pandemic. Science. 2020 Apr;368(6487):117.

29. Manyazewal T, Woldeamanuel Y, Blumberg HM, Fekadu A, Marconi VC. The fight to end tuberculosis must not be forgotten in the COVID-19 outbreak. Nat Med. 2020;26(6):811-2. 
30. Adepoju P. Tuberculosis and HIV responses threatened by COVID-19. Lancet HIV. 2020;7(5):e319-20.

31. YasriS, WiwanitkitV. Tuberculosis and novelWuhan coronavirus infection: pathological interrelationship. Indian J Tuberc. 2020;67(2):264.

32. Motta RC. DÁmbrosio L, García-García JM, Golettu D, Gualano G, Lipani F. Tuberculosis, COVID-19 and migrants: Preliminary analysis of deaths occuring in 69 patients from two cohorts. Pulmonol. 2020;26(4):23340.

33. Sharkie IK. BCG is a Good Immunotherapeutic Agent for Viral and Autoimmune Diseases: Is it a New Weapon against Coronavirus (COVID-19)? Electron J Gen Med. 2020;17(6):em229.

34. Rajarshi K, Chatterjee A, Ray S. BCG vaccination strategy implemented to reduce the impact of COVID-19: Hype or Hope? Med Drug Discov. 2020 Sep;7:100049

35. Redelman-Sidi G. Could BCG be used to protect against COVID-19? Nat Rev Urol. 2020;17(6):316-7.

36. Covián C, Retamal-Díaz A, Bueno SM, Kalergis AM. Could BCG Vaccination induce protective trained immunity for SARS-CoV-2? Front Immunol. $2020 ; 11: 970$
37. Hegarty PK, Sfakianos JP, Giannarini G, DiNardo AR, Kamat AM. COVID-19 and Bacillus Calmette-Guérin: what is the Link? Eur Urol Oncol. 2020;3(3):259-61.

38. Maciel EL, Gonçalves Júnior E, Dalcolmo MM. Tuberculosis and coronavirus: what do we know? Epidemiol Serv Saude. 2020 ;29(2):e2020128.

39. Amimo F, Lambert B, Magit A. What does the COVID-19 pandemic mean for HIV, tuberculosis, and malaria control? Trop Med Health. $2020 ; 48(1): 32$.

40. Rafael RMR, Netol M, Carvalho MMB, David HMSL, Acioli S, Faria MGA. Epidemiology, public policies and Covid-19 pandemics in Brazil: what can we expect? Rev enferm UERJ. 2020; 28:e49570. https://doi. org/10.12957/reuerj.2020.49570.

41. Wang ML, Behrman P, Dulin A, Baskin ML, Buscemi J, Alcaraz Kl, et al. Addressing inequities in COVID-19 morbidity and mortality: research and policy recommendations. Trans Behav Med 2020; 10(3): 516-19.

42. Stop TB. Partnership. The potential impact of the covid-19 response on tuberculosis in high-burden countries: a modelling analysis. Stop TB Partnership, Genève: Stop TB Partnership; 2020. [cited 2021 May 26]. Available from: http://www.stoptb.org/assets/documents/news/ Modeling\%20Report_1\%20May\%202020_FINAL.pdf 